\title{
Axis II comorbidity of borderline personality disorder: description of 6-year course and prediction to time-to-remission
}

\begin{abstract}
Zanarini MC, Frankenburg FR, Vujanovic AA, Hennen J, Reich DB, Silk KR. Axis II comorbidity of borderline personality disorder: description of 6-year course and prediction to time-to-remission.
\end{abstract} Acta Psychiatr Scand 2004: 110: 416-420. @ Blackwell Munksgaard 2004.

Objective: The purpose of this study was to compare the axis II comorbidity of 202 patients whose borderline personality disorder (BPD) remitted over 6 years of prospective follow-up to that of 88 whose BPD never remitted.

Method: The axis II comorbidity of 290 patients meeting both DIB-R and DSM-III-R criteria for BPD was assessed at baseline using a semistructured interview of demonstrated reliability. Over $96 \%$ of surviving patients were reinterviewed about their co-occurring axis II disorders blind to all previously collected information at three distinct follow-up waves: 2-, 4-, and 6-year follow-up.

Results: Both remitted and non-remitted borderline patients experienced declining rates of most types of axis II disorders over time. However, the rates of avoidant, dependent, and self-defeating personality disorders remained high among non-remitted borderline patients. Additionally, the absence of these three disorders was found to be significantly correlated with a borderline patient's likelihood-ofremission and time-to-remission; self-defeating personality disorder by a factor of 4 , dependent personality disorder by a factor of $3 \frac{1}{2}$, and avoidant personality disorder by a factor of almost 2 .

Conclusion: The results of this study suggest that axis II disorders cooccur less commonly with BPD over time, particularly for remitted borderline patients. They also suggest that anxious cluster disorders are the axis II disorders which most impede symptomatic remission from BPD.
M. C. Zanarini ${ }^{1}$, F. R. Frankenburg', A. A. Vujanovic ${ }^{1}$, J. Hennen ${ }^{1}$, D. B. Reich ${ }^{1}$, K. R. Silk ${ }^{2}$

${ }^{1}$ Laboratory for the Study of Adult Development, McLean Hospital, Belmont and the Department of Psychiatry, Harvard Medical School, Boston, MA and ${ }^{2}$ Department of Psychiatry, University of Michigan Medical School, Ann Arbor, MI, USA

Key words: borderline personality disorder; comorbidity; personality disorders

Doctor Mary C. Zanarini, McLean Hospital, 115 Mill Street, Belmont, MA 02478, USA.

E-mail: zanarini@mclean.harvard.edu

Accepted for publication April 7, 2004

\section{Introduction}

Cross-sectional studies have found that comorbid axis II disorders are common among borderline patients (1-4). Paranoid, avoidant, and dependent personality disorders are particularly common. However, only one follow-up study has systematically assessed co-occurring axis II disorders among borderline patients. Links et al. (5) administered a semistructured diagnostic interview for the DSM-III-R personality disorders for the first time at 7-year follow-up. These investigators found that remitted borderline patients were significantly less likely than non-remitted borderline patients to meet criteria for a number of other personality disorders, mostly anxious cluster disorders.

Aims of the study

The current study, which is the first study of a well-defined sample of borderline patients to 
systematically assess a full array of axis II disorders at four contiguous 2-year time periods, has two main aims. The first aim is to compare the rates of co-occurring axis II disorders of two groups of borderline patients: borderline patients who have and who have not achieved a remission from borderline personality disorder (BPD) at one or more of the study's follow-up periods. The second aim is to determine the relationship of these co-occurring disorders to time-to-remission from BPD.

\section{Material and methods}

Subjects

The current study is part of a multifaceted longitudinal study of the course of BPD - the McLean Study of Adult Development (MSAD). The methodology of this study has been described in detail elsewhere (6). Briefly, all subjects were initially inpatients at McLean Hospital in Belmont, Massachusetts who were admitted during a 3-year period (1992-1995). Each patient was screened to determine that he or she: (i) was between the ages of 18 and 35; (ii) had a known or estimated IQ of 71 or higher; (iii) had no history or current symptomatology of schizophrenia, schizoaffective disorder, bipolar I disorder, or an organic condition that could cause psychiatric symptoms; and (iv) was fluent in English.

\section{Procedures}

After the study procedures were explained at baseline, written informed consent was obtained. Each patient then met with a masters-level psychologist blind to the patient's clinical diagnoses. Three semistructured diagnostic interviews were administered: (i) the Structured Clinical Interview for DSM-III-R axis I disorders (SCID-I) (7), (ii) the Revised Diagnostic Interview for Borderlines (DIB-R) (8), and (iii) the Diagnostic Interview for DSM-III-R Personality Disorders (DIPD-R) (9). Excellent levels of interrater and test-retest reliability were achieved at baseline for both axis I and II disorders $(10,11)$.

At each follow-up wave, diagnostic information was assessed via interview methods similar to the baseline procedures by staff members blind to baseline diagnoses. After informed consent was obtained, our diagnostic battery was re-administered (a change version of the SCID-I, the DIB-R, and the DIPD-R). Excellent interrater reliability was maintained throughout the course of the study for both axis I and II diagnoses $(10,11)$.

\section{Statistical analyses}

Between-group comparisons involving categorical demographic data were computed by using the chi-square statistic corrected for continuity; between-group comparisons involving continuous demographic data were computed by using Student's $t$-test.

Data pertaining to axis II disorders were assembled in panel format (i.e. multiple records per patient, with one record for each assessment period for which data were available). Random effects regression modeling methods assessing the role of remission status and time, and controlling for clinically important baseline covariates [gender, race, age, socioeconomic status, Global Assessment of Functioning (GAF), and number of treatment modalities] were used in all analyses of axis II data (12). In this modeling work, probit analyses of binary dependent variables (e.g. avoidant personality disorder present/absent) were used. Interactions between remission status and time were checked in this modeling. Model fits were checked by examining partial residual plots. Because of the multiple comparisons involved in the analyses of axis II panel data, Bonferroni-type corrections were applied to the $P$-values for the main effects of remission status and time. As there were 15 such comparisons, this resulted in an adjusted $P$-value of $0.05 / 15=0.0033$.

We defined time-to-remission as the follow-up period at which remission was first achieved. Thus, possible values for this time-to-remission measure were 1,2 , or 3 , with time $=1$ for persons first achieving remission at the first follow-up period (at 24 months postbaseline), time $=2$ for persons first achieving remission at the second follow-up period (at 48 months postbaseline), time $=3$ for persons first achieving remission at the third follow-up period (at 72 months postbaseline). Because this time measure is discrete, survival analytic methods assuming continuous time (such as Cox proportional hazards regression modeling) could not be employed. Instead, we used discrete time-to-event modeling methods for axis II disorders, which yield adjusted hazard ratios (and 95\% confidence intervals) (13).

\section{Results}

A total of 290 patients met both DIB-R and DSMIII-R criteria for BPD. In the follow-up waves, 275 borderline patients were reinterviewed at 2 years, 269 at 4 years, and 264 at 6 years. By this time, 26 borderline patients were no longer in the study: 11 had committed suicide, three others died of natural 
Table 1. Baseline demographic characteristics of remitted and non-remitted borderline patients

\begin{tabular}{|c|c|c|c|c|c|c|}
\hline \multirow[b]{2}{*}{ Baseline characteristic } & \multicolumn{2}{|c|}{$\begin{array}{c}\text { Remitted borderline } \\
\text { personality disorder (BPD) } \\
\qquad(N=202)\end{array}$} & \multicolumn{2}{|c|}{$\begin{array}{l}\text { Non-remitted BPD } \\
\qquad(N=88)\end{array}$} & \multirow[b]{2}{*}{$\chi^{2}$-value (d.f. $=1$ ) } & \multirow[b]{2}{*}{$P$-level } \\
\hline & $N$ & $\%$ & $N$ & $\%$ & & \\
\hline Female & 161 & 79.9 & 72 & 81.8 & 0.17 & 0.67 \\
\hline White & 173 & 85.6 & 80 & 90.9 & 1.53 & 0.22 \\
\hline Baseline characteristic & Mean & SD & Mean & SD & $t$-test (d.f. $=288$ ) & P-level \\
\hline Age & 26.6 & 5.8 & 27.8 & 5.5 & 1.60 & 0.11 \\
\hline $\begin{array}{l}\text { Socioeconomic status } \\
\qquad(1=\text { highest, } 5=\text { lowest })\end{array}$ & 3.2 & 1.5 & 3.7 & 1.4 & 2.57 & 0.01 \\
\hline $\begin{array}{l}\text { Global Assessment } \\
\text { of Functioning (GAF) }\end{array}$ & 39.8 & 7.3 & 36.8 & 7.6 & 3.19 & 0.002 \\
\hline Number of prior treatment modalities & 4.7 & 2.1 & 5.4 & 2.0 & 2.77 & 0.007 \\
\hline
\end{tabular}

causes, nine discontinued their participation, and three were lost to follow-up. All told, over $96 \%$ of surviving borderline patients were reinterviewed at all three follow-up waves.

Table 1 shows the demographic characteristics of the 202 ever-remitted borderline patients and the 88 never-remitted borderline patients. (Remission was defined as no longer meeting DIB-R and DSM-III-R criteria for BPD for at least 2 years.) As can be seen, the two groups were very similar in age, race, and gender. However, remitted borderline patients were from a significantly higher socioeconomic background than non-remitted borderline patients, had a significantly higher GAF score, and had participated in a significantly smaller number of forms of psychiatric treatment prior to their index admission. It is important to note that while these statistically significant differences emerged from the data, they represent clinically very modest differences - both groups of patients came from lower-middle class backgrounds, were very impaired in their overall functioning, and had had multiple forms of psychiatric treatment.

Table 2 shows the comorbid axis II disorders of the ever-remitted borderline patients and the neverremitted borderline patients. At the very stringent Bonferroni-corrected $P$-level of 0.0033 , a significantly higher percentage of never-remitted borderline patients than ever-remitted borderline patients met criteria for an anxious cluster and a non-BPD dramatic cluster disorder. In terms of specific disorders, a significantly higher percentage of never-remitted borderline patients than ever-remitted borderline patients met criteria for avoidant, dependent, self-defeating, histrionic, and narcissistic personality disorder. There were no significant between-group differences found for odd cluster disorders. When all subjects were considered together, the rates of 12 of the 15 disorders studied declined significantly over time. The exceptions were schizoid, schizotypal, and sadistic personality disorder.

We next assessed time to remission for borderline subjects who did not meet criteria at one or more follow-up periods for the five disorders that were found to be significant in the previous set of analyses: avoidant, dependent, self-defeating, histrionic, and narcissistic personality disorder. In terms of time-to-remission, $47.0 \%(n=95)$ of the 202 borderline patients who experienced a remission of their BPD first remitted by 2-year follow-up, $26.7 \%(n=54)$ first remitted by 4 -year follow-up, and $26.3 \%(n=53)$ first remitted by 6-year follow-up (6). Table 3 shows the hazard ratios for the disorders from this set that were found to be significant in these analyses. As can be seen, the absence of any of these three disorders was significantly correlated with occurrence-ofremission and time-to-remission; self-defeating personality disorder by a factor of 4 , dependent personality disorder by a factor of $3 \frac{1}{2}$, and avoidant personality disorder by a factor of almost 2 .

\section{Discussion}

Two main findings have emerged from this study. The first is that most co-occurring personality disorders declined significantly over time. This substantial decline was noted in many cases for both remitted and non-remitted borderline patients. The three exceptions were avoidant, dependent, and self-defeating personality disorders, which remained common among non-remitted borderline patients. Even by the 5-6 years after their index admission, 59\% of non-remitted borderline patients met criteria for avoidant personality disorder, $45 \%$ for dependent personality disorder, and $27 \%$ for self-defeating personality disorder. This contrasts with $16 \%, 8 \%$, and $1 \%$ respectively for remitted borderline patients. This finding of higher rates of these three disorders 
Table 3. Proportional hazard ratios of time-to-remission for absence of axis ॥ disorders experienced by borderline patients

Absence of disorder Hazard ratio SE $z$-score $P$-level $95 \%$ confidence interval

\begin{tabular}{lrrrrr}
\hline Avoidant & 1.95 & 0.39 & 3.330 & 0.001 & $1.32-2.88$ \\
Dependent & 3.49 & 0.86 & 5.068 & $<0.001$ & $2.15-5.67$ \\
Self-defeating & 4.06 & 1.72 & 3.315 & 0.001 & $1.77-9.30$ \\
\hline
\end{tabular}

among non-remitted than remitted borderline patients is consistent with the results of the crosssectional study conducted by Links et al. (5). The finding that rates of axis II disorders decline over time is consistent with the results of other studies that have found that axis II disorders tend to remit over time $(14,15)$. The fact that they do so suggests that the current definition of a personality disorder as a chronic condition should be modified in official systems of nomenclature to a condition that is slow to change.

The second main finding concerns the predictive power of the absence of various disorders to timeto-remission from BPD. Only anxious cluster disorders were found to be significant in these analyses. The absence of avoidant, dependent, and self-defeating personality disorders significantly reduced a borderline patient's time to remission or looked at another way, significantly improved a borderline patient's chances of remitting.

It may be that borderline patients with the added burden of one or more of these 'passive' temperaments or aspects of temperament have less emotional energy or determination to put into the struggle to recover from BPD than patients with more active or assertive temperaments. However, this does not explain why the rates of avoidant, dependent, and self-defeating personality disorders remained relatively constant for non-remitted borderline patients, while declining sharply for remitted borderline patients. It might be that for non-remitted borderline patients these co-occurring disorders represent enduring aspects of their temperament, while for remitted borderline patients they were symptomatic manifestations secondary to their BPD. If so, once their borderline psychopathology was significantly diminished, their fear of embarrassment and rejection, dependency, and masochism also may have declined in severity. In other words, there may be subtypes of borderline patients and those most likely to remit in the short- to mid-term are less temperamentally impaired than those whose borderline psychopathology remains relatively constant. This finding suggests that treatments aimed at these different subtypes of BPD need to be developed.

As a final note, it seems unlikely that these differences in axis II psychopathology were due to 


\section{Zanarini et al.}

treatment as a significantly higher percentage of non-remitted than remitted borderline patients reported receiving both out-patient and more intensive treatment (psychiatric hospitalization, residential care, day treatment) over time (16). Even 5-6 years after their index admission, 100\% of non-remitted borderline patients were in some form of out-patient treatment and $77 \%$ were in some form of more intensive treatment. For remitted borderline patients, the comparable figures were $84 \%$ and $24 \%$ respectively.

\section{Limitations and directions for further research}

The main limitation of this study is that all of the borderline patients were initially in-patients. It is not clear if less severely disturbed borderline patients would exhibit these same patterns of comorbidity. In addition, the current study assessed DSM-III-R axis II psychopathology and this might be quite different from DSM-IV axis II comorbidity because of changes in a number of the criteria sets (and the elimination of self-defeating and sadistic personality disorders). One might also wonder how dependent the findings of the current study are on our definition of remission as no longer meeting criteria for the disorder for a period of 2 years or more. However, another study using a different definition of remission has found consistent findings in two shorter term studies $(14,15)$.

Further research of this kind based on DSM-IV criteria for axis II disorders is needed. Further research is also needed to determine if there are, as we suggest, temperamental and symptomatic subtypes of BPD.

\section{Conclusions}

Taken together, the results of this study suggest that axis II psychopathology decreases significantly over time for borderline patients, particularly remitted borderline patients. They also suggest that anxious cluster disorders are the axis II disorders most strongly associated with the failure to remit from BPD.

\section{Acknowledgements}

This research was supported by NIMH grants MH47588 and MH62169.

\section{References}

1. McGlashan TH, Grilo CM, Skodol AE et al. The collaborative longitudinal personality disorders study: baseline axis I/II and II/II diagnostic co-occurrence. Acta Psychiatr Scand 2000;102:256-264.

2. Nurnberg HG, Raskin M, Levine PR, Pollack S, Siegel O, PRINCE R. The comorbidity of borderline personality disorder and other DSM-III-R axis II personality disorders. Am J Psychiatry 1991;148:1371-1377.

3. Oldham JM, Skodol AE, Kellman HD, Hyler SE, Rosnick L, DAviES M. Diagnosis of DSM-III-R personality disorders by two structured interviews: patterns of comorbidity. Am J Psychiatry 1992;149:213-220.

4. Zanarini MC, Frankenburg FR, Dubo ED et al. Axis II comorbidity of borderline personality disorder. Compr Psychiatr 1998;39:296-302.

5. Links PS, Heslegrave R, Van Reekum R. Prospective followup study of borderline personality disorder: prognosis, prediction of outcome, and axis II comorbidity. Can J Psychiatry 1998;43:265-270.

6. Zanarini MC, Frankenburg FR, Hennen J, Silk KR. The longitudinal course of borderline psychopathology: 6-year prospective follow-up of the phenomenology of borderline personality disorder. Am J Psychiatry 2003;160:274-283.

7. Sitzer RL, Williams JB, Gibbon M, First M. Structured Clinical Interview for DSM-III-R (SCID): I. History, rational, and description. Arch Gen Psychiatry 1992; 49:624-629.

8. Zanarini MC, Gunderson JG, Frankenburg FR, Chauncey DL. The Revised Diagnostic Interview for Borderlines: discriminating BPD from other axis II disorders. J Pers Disord 1989;3:10-18.

9. Zanarini MC, Frankenburg FR, Chauncey DL, Gunderson JG. The Diagnostic Interview for Personality Disorders: interrater and test-retest reliability. Compr Psychiatry 1987;28:467-480.

10. Zanarini MC, Frankenburg FR, Vujanovic AA. The interrater and test-retest reliability of the Revised Diagnostic Interview for Borderlines (DIB-R). J Pers Disord 2002; 16:270-276.

11. Zanarini MC, Frankenburg FR. Attainment and maintenance of reliability of axis I and II disorders over the course of a longitudinal study. Compr Psychiatry 2001;42:369374.

12. Greene WH. Econometric analysis, 4 th edn. Upper Saddle River, NJ: Prentice hall, 2000:212-237.

13. Allison PD. Event history analysis: regression for longitudinal event data. Newburg Park, CA: Sage, 1984:14-22.

14. Shea MT, Stout R, Gunderson J et al. Short-term diagnostic stability of schizotypal, borderline, avoidant, and obsessive-compulsive personality disorders. Am J Psychiatry 2002;159:2036-2041.

15. Grillo CM, Shea MT, SAnislow CA et al. Two-year stability and change in schizotypal, borderline, avoidant, and obsessive-compulsive personality disorders. J Consult Clin Psychol (in press).

16. Zanarini MC, Frankenburg FR, Hennen J, Silk KR. Mental health service utilization of borderline patients and axis II comparison subjects followed prospectively for six year. J Clin Psychiatry 2004;65:28-36. 